\title{
Adaptação Brasileira do Questionário de Esquemas de Young - Versão Breve (YSQ-S3)
}

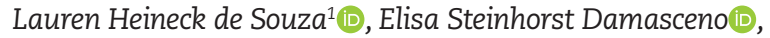 \\ Felipe Gonçalves Ferronatto(D), Margareth da Silva Oliveira(D) \\ Pontifícia Universidade Católica do Rio Grande do Sul, Porto Alegre-RS, Brasil
}

\section{RESUMO}

Este estudo tem como objetivo descrever o processo de adaptação semântica do Questionário de Esquemas de Young - versão breve (YSQ-S3) para o português brasileiro e apresentar dados preliminares da consistência interna do instrumento. Trata-se de um estudo instrumental que seguiu os passos de tradução, síntese, avaliação por experts, avaliação pelo público-alvo, retrotradução e estudo piloto. Inclui uma etapa de estudo de evidências preliminares, na qual foram aplicados o YSQ-S3 e uma ficha de dados sociodemográficos com 200 participantes para análise da consistência interna utilizando alfa de Cronbach. Os resultados indicam que o instrumento apresenta boa adequação semântica para uso no Brasil. A consistência interna total foi classificada como excelente. Destaca-se a importância do seguimento de estudos de evidências de validade.

Palavras-chave: questionário de esquemas de Young; terapia do esquema; adaptação; psicometria; confiabilidade.

\section{ABSTRACT - Brazilian adaptation of the Young Schema Questionnaire - Short Form (YSQ-S3)}

This study aimed for the semantic adaptation of the Young Schema Questionnaire - short form (YSQ-S3) to Brazilian Portuguese and presents preliminary data on the internal consistency of the instrument. It was an instrumental study that followed the steps of translation, synthesis, expert and focus group evaluation, back-translation and pilot study. It included a preliminary evidence study stage, in which the YSQ-S3 and a sociodemographic data sheet with 200 participants were analyzed for internal consistency with Cronbach's alpha. The results indicate that the Brazilian version of the YSQ-S3 presents good semantic adequacy. Overall internal consistency was rated as excellent. A validation study of this version is currently being carried out.

Keywords: Young Schema Questionnaire; Schema Therapy; Adaptation; Psychometry; Reliability.

\section{RESUMEN - Adaptación brasileña del Young Scheme Questionnaire - Versión Breve (YSQ-S3)}

Este estudio tiene como objetivo describir el proceso de adaptación semántica del Young Scheme Questionnaire - versión breve (YSQ-S3) para el idioma brasileño y presentar datos preliminares de la consistencia interna del instrumento. Se trata de un estudio instrumental que siguió los pasos de la traducción, síntesis, evaluación por los expertos, evaluación del público objetivo, retrotraducción y estudio piloto. Incluye una etapa de estudio de evidencias preliminares en las que se aplicaron el YSQ-S3 y una ficha de datos sociodemográficos con 200 participantes para el análisis de la consistencia interna a partir del alfa de Cronbach. Los resultados indicaron que el instrumento presenta buena adecuación semántica para uso en Brasil. La consistencia interna total fue clasificada como excelente. Se destaca la importancia del seguimiento de estudios de evidencias de validez.

Palabras clave: Cuestionario de Esquemas de Young; Terapia del Esquema; Adaptación; Psicometría; Confiabilidad.

A Terapia do Esquema - TE (Young, 1990; Young, Klosko, \& Weishaar, 2003) foi desenvolvida para o tratamento de transtornos de personalidade e de indivíduos com sintomas crônicos. Atualmente, a abordagem se expande enquanto recurso terapêutico integrativo para compreensão e mudança de padrões cognitivos, emocionais e comportamentais. Há uma crescente popularidade da abordagem nos últimos 10 anos (Van Vreeswijk, Broersen, \& Nadort, 2012) e estudos vêm demonstrando promissora aplicabilidade com transtornos de personalidade e demais transtornos psicológicos, como os de ansiedade e depressão (Jacob \& Arntz, 2013; Hawke \& Provencher, 2011; Taylor, Bee, \& Haddock, 2016).

A TE é uma abordagem psicoterápica integrativa com base na Terapia Cognitiva, em composição com elementos e concepções comportamentais, de apego, relações objetais, construtivismo, psicanálise, Gestalt e terapia focada na emoção (Edwards \& Arntz, 2012; Young

${ }^{1}$ Endereço para correspondência: Grupo de Pesquisa (GAAPCC). Avenida Ipiranga, 6681, sala 941, Partenon, 90619-900, Porto Alegre, RS. Tel.: (51) 3320-3500, ramal 7749. E-mail: lauren_heineck@hotmail.com

Artigo derivado da Dissertação de Mestrado de Lauren Heineck de Souza com orientação de Margareth da Silva Oliveira, defendida em 2019 no Programa de Pós-Graduação em Psicologia da Pontifícia Universidade Católica do Rio Grande do Sul. Título da dissertação: "Adaptação e validação do questionário de esquemas de Young: versão breve (YSQ-S3) para uso no Brasil". 
et al., 2003). Nessa perspectiva, parte-se da compreensão dos impactos do desenvolvimento humano e das experiências na formação da identidade, psicopatologias e noções de vida do sujeito (Young et al., 2003). Esse modelo possibilita uma compreensão ampliada da personalidade, que parte do princípio que as necessidades emocionais não supridas ao longo da infância e adolescência levam ao desenvolvimento de Esquemas Iniciais Desadaptativos (EIDs - Young, 1990; Young et al., 2003).

O termo "esquema", nesse contexto, refere-se a um padrão amplo, difuso e duradouro, formado ao longo da vida e que interfere na percepção que o sujeito tem sobre a realidade e nos seus padrões comportamentais. Os EIDs são formados por memórias, emoções e sensações corporais vivenciadas especialmente na infância e adolescência e carregam influências importantes das relações com pares e cuidadores (Young et al., 2003).

Entende-se que, para ter um desenvolvimento psicológico saudável, é indispensável que a interação entre o ambiente e o temperamento do indivíduo supra suas necessidades emocionais básicas (Lockwood \& Perris, 2012; Young et al., 2003). Essas necessidades envolvem a percepção de vínculo seguro com outros indivíduos, possibilidade de desenvolver autonomia e sentido de identidade, validação das expressões emocionais, direito à espontaneidade e lazer e a limites realistas. A não satisfação dessas necessidades pode acarretar no desenvolvimento de EIDs (Lockwood \& Perris, 2012; Young et al., 2003).

Conforme modelo proposto por Young et al. (2003), são considerados 18 EIDs, agrupados em cinco domínios. O $1^{\circ}$ domínio "Desconexão e rejeição" se refere ao conjunto de esquemas relacionados a dificuldades de formar vínculo seguros e estáveis com os outros. Envolve a expectativa do indivíduo de que não the serão supridas as necessidades básicas de afeto, proteção, segurança, empatia, cuidado e estabilidade. A família de origem costuma ser caracterizada como ser fria, distante, rejeitadora, imprevisível, impaciente ou abusiva. Os cinco EIDs desse domínio são: Abandono/ Instabilidade; Desconfiança/Abuso; Privação emocional; Defectividade/Vergonha e Isolamento social/ Alienação (Young et al., 2003).

$\mathrm{O} 2^{\circ}$ domínio "Autonomia e desempenho prejudicados" está relacionado à dificuldade do indivíduo de reconhecer sua capacidade de viver de forma autônoma ou ter um bom desempenho. A presença de esquemas desse domínio costuma acarretar em problemas para se diferenciar das figuras paternas e maternas para funcionar com independência, sentem-se demasiadamente vulneráveis e dependentes dos outros inclusive para tarefas diárias. A família de origem geralmente é superprotetora, emaranhada ou excessivamente crítica, fornece poucas oportunidades de exploração e autonomia que incentivem a criança e favoreçam um senso adequado de autoconfiança. Esse domínio inclui os EIDs: Dependência/
Incompetência; Vulnerabilidade ao dano ou à doença; Emaranhamento/Self subdesenvolvido e Fracasso (Young et al., 2003).

No $3^{\circ}$ domínio, "Limites prejudicados", os indivíduos não internalizaram limites adequados para autodisciplina ou senso de reciprocidade e respeito aos diretos dos outros. Muitas vezes têm dificuldades para cumprir metas e realizações pessoais de longo prazo, tolerar frustrações ou respeitar os compromissos com as outras pessoas. Em geral, a família de origem foi excessivamente permissiva ou indulgente, não favorecendo orientações, limites, tolerância a possíveis desconfortos ou senso de reciprocidade. Os EIDs desse domínio são: Arrogo/grandiosidade e Autocontrole/autodisciplina insuficientes (Young et al., 2003).

O $4^{\circ}$ domínio, "Orientação para o outro", é caracterizado por excessivo atendimento das necessidades dos outros em detrimento das próprias necessidades do paciente. Em geral, esse direcionamento ao outro tem como objetivo receber aprovação, evitar retaliações e manter as conexões emocionais, o que pode levar o paciente a suprimir suas emoções e desejos. A família de origem geralmente ensinou que o amor e a aceitação são condicionais, levando a criança a buscar suprir essas necessidades atendendo às expectativas, muitas vezes irrealistas, dos membros desse grupo. Esse domínio é composto por: Subjugação; Autossacrifício e Busca de aprovação/Busca de reconhecimento (Young et al., 2003).

O $5^{\circ}$ domínio, "Supervigilância e inibição", tem como aspecto central a ênfase excessiva na inibição de sentimentos e espontaneidade, ou no seguimento de regras rígidas sobre desempenho e ética à custa do relaxamento, felicidade, prazer, autoexpressão, saúde e relacionamento íntimos. A origem na infância geralmente está relacionada à repressão, perfeccionismo, rigidez ou punição. Há constante preocupação com a possibilidade de ocorrência de eventos negativos ou erros e sensação que haverá uma catástrofe caso haja falhas na vigilância. Inclui os esquemas: Negativismo/Pessimismo; Inibição emocional; Padrões inflexíveis/Postura crítica exagerada e Postura punitiva (Young et al., 2003).

A distribuição de EIDs em domínios vem sendo investigada em diversos estudos (Bach, Simonsen, Christoffersen, \& Kriston, 2017a; Calvete, Orue, \& González-Diez, 2013; Soygüt, Karaosmanoğlu, \& Cakir, 2009; Kriston, Schäffer, Jacob, Härter, \& Hölzel, 2013; Sakulsriprasert, Phukao, Kanjanawong, \& Meemon, 2016; Saritas \& Gençöz, 2011; Schmidt, Joiner, Young, \& Telch, 1995; Lavergne, Marie, \& Mehran, 2015). O pacote oficial da Terapia do Esquema adquirido junto ao International Society of Schema Therapy (ISST) inclui a proposta de um modelo que contempla quatro Clusters de ordem superior (Young, 2014).

Nesse modelo, é apresentada a seguinte distribuição: $1^{\circ}$ Cluster - Privação emocional, Isolamento social/ Alienação, Inibição emocional, Defectividade/Vergonha, 
Desconfiança/Abuso e Negatividade/Pessimismo; $2^{\circ}$ Cluster - Dependência/Incompetência, fracasso, abandono/Instabilidade, emaranhamento/Self subdesenvolvido e Vulnerabilidade ao dano ou doença; $3^{\circ}$ Cluster - Arrogo/ Grandiosidade, Autocontrole/Autodisciplina insuficientes e Busca de aprovação/Reconhecimento; $4^{\circ}$ Cluster - Autossacrifício, padrões inflexíveis/Postura crítica exagerada e Postura punitiva; "Esquemas sem classificação" - Busca de aprovação/Reconhecimento; Negatividade/ Pessimismo e Postura punitiva (Young, 2014; Bach, Lockwood, \& Young, 2017b).

Para auxiliar na avaliação, identificação, pesquisa e trabalho com EIDs, foi desenvolvido o Young Schema Questionnaire (Young, 1990). Trata-se de um questionário preenchido a partir de autorrelato, os escores são mensurados a partir de 90 itens com escala tipo Likert com pontuações de 1 a 6 pontos $(1=$ Completamente falso sobre mim e $6=$ Me descreve perfeitamente).

Inicialmente, o YSQ foi desenvolvido para avaliar 16 EIDs a partir de 205 itens (Young \& Brown, 1990). Após estudos de análise fatorial (Schmidt et al., 1995), foi elaborada uma versão breve, o YSQ-S2 (Young \& Brown, 1999), com 75 itens que avalia 15 EIDs, todos os descritos acima com exceção de Busca de aprovação/ Reconhecimento, Negatividade/Pessimismo e Postura punitiva. Esta versão do instrumento foi adaptada para uso no Brasil, apresenta evidências de validade concorrente quando comparado com a Escala fatorial de ajustamento emocional/neuroticismo e boa consistência interna geral $(\alpha=0,95)$. As confiabilidades avaliadas de acordo com os EIDs foram satisfatórias, variando de 0,72 a 0,90 , com exceção de Dependência/Incompetência, cujo alpha foi 0,57 (Cazassa \& Oliveira, 2012).

Mais recentemente, foi desenvolvida a terceira versão do instrumento, disponível na versão longa (YSQ-L3 - Young, 2003), com 232 itens e, na versão breve, com 90 itens (YSQ-S3 - Young, 2005). O YSQ-S3, instrumento foco deste estudo, mensura a ativação dos 18 EIDs, está adaptado para uso em diversos países e estudos vêm investigando suas propriedades psicométricas. Algumas versões investigadas são a romena (Trip, 2006), turca (Soygüt, Karaosmanoğlu, \& Cakir, 2009; Saritas, \& Gençöz, 2011), portuguesa (Portugal; Rijo \& Gouveia, 2008), finlandesa (Saariaho, Saariaho, Karila, \& Joukama, 2009), palestina (Alfasfos, 2009), franco-canadense (Hawke, \& Provencher, 2012), espanhola (Calvete et al., 2013), alemã (Kriston et al., 2013), grega (Lyrakos, 2014), coreana (Lee, Choi, Rim, Won, \& Lee, 2015), francesa (Lavergne, Marie, \& Mehran, 2015), tailandesa (Sakulsriprasert et al., 2016), dinamarquesa (Bach et al., 2017b) e polonesa (Oettingen, Chodkiewicz, Macik, \& Gruszcyńzka, 2017).

É essencial que instrumentos de avaliação sejam adequadamente adaptados para a realidade na qual serão utilizados (Borsa, Damásio, \& Bandeira, 2012). Tendo em vista a relevância do instrumento YSQ-S3 para avaliação de EIDs, destaca-se a importância do instrumento para uso clínico e em pesquisas no Brasil. A adaptação semântica consiste adaptar o instrumento considerando os aspectos linguísticos, coerência com o idioma, com o construto e o contexto cultural no qual será aplicado (Hambleton, 2005). Para além da tradução, esse processo considera a manutenção do significado do conteúdo dos itens, características psicométricas e adequação à população-alvo (Cassepp-Borges, Balbinotti, \& Teodoro, 2010).

A área de avaliação psicológica envolve a operacionalização de teorias e construtos psicológicos para observação prática desses elementos. Essa operacionalização permite avaliação de processos psicológicos e evoluções de modelos teóricos explicativos (Primi, 2003). A adequada avaliação demanda instrumentos adaptados de forma rigorosa e estruturada, pautados em processos claramente descritos, aporte teórico consistente e evidências empíricas (Primi, 2010).

Considerando a importância do YSQ-S3 para identificação de EIDs em pesquisas e na prática clínica, este estudo tem como objetivo descrever o processo de adaptação semântica do YSQ-S3 para o português brasileiro. Além disso, serão apresentados dados preliminares da consistência interna do instrumento.

\section{Método}

\section{Primeira Etapa: Tradução e Adaptação Transcultural}

A obtenção dos direitos para tradução e adaptação da versão oficial do YSQ-S3 foi feita em contato com o ISST. A autorização recebida garante direitos completos de uso e distribuição das traduções oficiais dos questionários para o português brasileiro. No documento, é destacada a exclusividade da versão para uso no Brasil. Após a obtenção dessa autorização formal, foi feita a aquisição do Pacote de Questionários da Terapia do Esquema. Dessa forma, os processos de tradução e adaptação semântica do instrumento seguiram as orientações e exigências do documento. Os processos exigidos pelo ISST incluem Adaptação (1), Revisão (2) e Retrotradução (5). Além disso, foram inclusas etapas de Avaliação da Síntese por Experts (3), Avaliação pelo Público-Alvo (4) e Estudo Piloto (6) (Borsa et al., 2012).

A primeira etapa (1) consistiu na tradução e adaptação do instrumento do inglês para o português brasileiro, realizada por um nativo do português brasileiro, com inglês fluente e familiarizado com a Terapia do Esquema. Após a conclusão do primeiro processo, a versão traduzida passou pelo processo de revisão (2) por um segundo nativo do português brasileiro também familiarizado com a Terapia do Esquema. O revisor fez sugestões de mudanças e, posteriormente, tradutor e revisor se reuniram para avaliar os itens e fazer as modificações necessárias até chegar a uma síntese. A versão oriunda do 
consenso entre tradutor e revisor seguiu para as próximas etapas de adaptação (Borsa et al., 2012).

A síntese foi encaminhada para avaliação de três juízes experts na área (3). Foi solicitada a avaliação da qualidade da introdução e dos itens considerando clareza, tradução e regionalismo (Borsa et al., 2012). Para avaliação, foram fornecidas escolhas "sim" e "não", com espaço para comentários adicionais. Para cálculo da concordância entre juízes, foi utilizada a porcentagem de concordância absoluta, que consiste em calcular o número de vezes que os juízes concordaram e dividir pelo total de itens. Considera-se a concordância aceitável acima de $75 \%$ e alta a partir de $90 \%$, segundo Stemler (2004). Já os comentários dos experts foram observados e alguns itens foram alterados.

$\mathrm{Na}$ etapa de Avaliação pelo Público-Alvo (4), o instrumento foi aplicado em um grupo focal com oito estudantes universitários, sendo cinco do sexo feminino e três do masculino, com média de idade de 23,25 anos $(D P=2,76)$, residentes no Rio Grande do Sul. Essa fase teve como objetivo avaliar a compreensão dos itens e das instruções, bem como verificar necessidades de ajustes. O procedimento consistiu na leitura em grupo do questionário e, a cada item, foi verificada a compreensão dos participantes e adequação das expressões utilizadas. As falas foram registradas e posteriormente analisadas.

A Retrotradução (5) foi feita por um tradutor profissional nativo da língua inglesa, que também possui fluência no português brasileiro. Nesse processo, é realizada a tradução reversa da versão final em português para o inglês sem consultar as versões originais. A retrotradução foi enviada ao ISST e essa etapa foi finalizada.

Com a versão final, foi aplicado o Estudo Piloto (6), que consiste numa aplicação preliminar com uma amostra pequena de participantes de população geral para avaliar a necessidade de ajustes e possíveis dificuldades de compreensão. Participaram dessa etapa 10 sujeitos, de diferentes classes sociais, idades e níveis de escolaridade por conveniência para avaliar a compreensão dos itens. Após aplicação do questionário, os participantes foram questionados sobre a clareza dos itens e se houve dúvidas durante o preenchimento.

\section{Segunda Etapa: Avaliação Preliminar de Propriedades Psicométricas}

A versão final do instrumento foi aplicada com o intuito de avaliação preliminar das propriedades psicométricas. Participaram dessa etapa do estudo, 200 sujeitos de população geral com coleta on-line.

\section{Instrumentos}

Para caracterizar os participantes, foi utilizada uma ficha de dados sociodemográficos. Para o estudo do instrumento, foi aplicado o Questionário de Esquemas de Young - versão breve (YSQ-S3), conforme descrito anteriormente.

\section{Procedimentos}

Os participantes foram recrutados por conveniência a partir das redes sociais dos pesquisadores e seus conhecidos. Os sujeitos foram convidados para a pesquisa e, após a leitura e compreensão do Termo de Consentimento Livre e Esclarecido (TCLE), iniciaram a participação preenchendo os instrumentos on-line no Qualtrics Survey Software.

O presente estudo está inserido em um projeto maior de adaptação e validação dos Questionários da Terapia do Esquema para o Brasil e possui aprovação do Comitê de Ética em Pesquisa da Pontifícia Universidade Católica do Rio Grande do Sul (PUCRS), CAEE: 80925517.0.0000.5336. Foram respeitados os princípios éticos da Resolução no 466/12 do Conselho Nacional de Saúde.

\section{Análise dos Dados}

Os dados oriundos da coleta com os participantes foram tabulados e analisados utilizando o Statistical Package for the Social Sciences - SPSS 24. Para caracterização da amostra, foram utilizadas análises descritivas e de frequências. Para avaliação da consistência interna, foi utilizada a análise de confiabilidade com coeficiente de alfa de Cronbach, considerando satisfatório $\alpha \geq 0,7$.

\section{Resultados}

\section{Primeira Etapa: Tradução e Adaptação Transcultural}

\section{Tradução, Revisão e Síntese}

A versão final do Young Schema Questionnaire - Short Form, 3rd Edition para o português brasileiro foi intitulada Questionário de Esquemas de Young - Versão Breve (YSQ-S3). O instrumento apresenta os mesmos 90 itens na ordem da versão original. $\mathrm{Na}$ etapa de adaptação semântica, alguns itens necessitaram de modificações linguísticas para manutenção da coerência dos itens com o conteúdo subjacente e facilitar a compreensão dos respondentes no Brasil (Tabela 1).

No item 1 (I haven't had someone to nurture me, share $\mathrm{him} / \mathrm{her} \mathrm{self} \mathrm{with} \mathrm{me,} \mathrm{or} \mathrm{care} \mathrm{deeply} \mathrm{about} \mathrm{everithing} \mathrm{that} \mathrm{happens}$ to me) o termo "nurture me" foi substituído pelos termos equivalentes "me dar afeto, cuidado e proteção". No item 7 (I do not feel capable of getting by on my own in everyday life), o termo "getting by on my own" foi adaptado para a expressão coloquial “"me virar' sem ajuda dos outros" com o intuito de mantê-la alinhada ao esquema de Dependência/ Incompetência. No item 8 (I can't seem to escape the feeling that something bad is about to happen), a expressão "escape the feeling" foi adaptada para "parar de sentir", considerando que o verbo "fugir", tradução do termo original "esca$p e$ ", não é comumente seguido de substantivo abstrato no português brasileiro, optou-se pela substituição deste 
pelo termo "deixar de", transmitindo a ideia de interrupção do sentimento que o item propõe.

No item 10 (I think that if I do what I want, I'm only asking for trouble), foi feita importante reformulação. Originalmente, esse item apresenta "I'm only asking for trouble", que foi adaptado para "os outros não me apoiarão e poderão ficar bravos ou chateados comigo". Essa modificação foi feita com o intuito de manter coerência com o esquema de Subjugação e tendo em vista o pouco uso da expressão original no Brasil. No item 17 (Even when things seem to be going well, I feel that it is only tempora$r y$ ), foi acrescentado "para que elas comecem a dar errado". Esse acréscimo foi feito com o intuito de possibilitar melhor compreensão e reduzir as possibilidades de não entendimento da expressão "I feel that it is only temporary" e tendo em vista que esse item avalia o EID Negativismo/ Pessimismo. Para facilitar a compreensão do item 28, foram realizadas alterações na ordem dos verbos na frase "or else they will retaliate, get angry, or reject me in some way" para que os complementos verbais necessários no português não alterassem a cadência da frase, comprometendo sua clareza.

No item 32 (I'm special and shouldn't have to accept many of the restrictions or limitations placed on other people), que mensura merecimento/grandiosidade, o adjetivo "special" foi adaptado para "diferenciado" a partir da compreensão de que a tradução literal do termo para o português, que seria "especial", poderia ser interpretada como "único" ou "singular", e que esses são atributos frequentemente associados à ideia de um indivíduo "não idêntico", ao invés de um indivíduo "melhor", como o sugere o item original em inglês. A expressão "tenho que me dar mal mesmo" foi utilizada enquanto equivalente "I should expect to lose out" no item 36 (If I don't try my hardest, I should expect to lose out). Ainda que se trate de uma expressão coloquial, foi escolhida dessa forma com o intuito de possibilitar maior acesso ao esquema de Postura punitiva, tendo em vista as expressões utilizadas no Brasil para representar o sentido e intensidade da frase original.

No item 59 (I feel that I'm not lovable), que mensura Defectividade, a expressão "I'm not lovable" foi adaptada para "Sou alguém que não pode ser amado". Em português, o termo "amável" tem também o significado de "gentil, simpático, delicado, afável”, o que possibilitaria uma interpretação dupla e poderia descaracterizar o item. Para facilitar a compreensão da expressão abstrata "vivessem através de mim" no item 63 (I often feel as IF my parent(s) are living through me - that I don't have a life of my own), que mensura esquema de Emaranhamento, foi acrescentada a sentença "meus pais vivessem a minha vida".

No item 85 (I can't let myself off the hook easily or make excuses for my mistakes), a expressão "I can't let myself off the hook easily" foi adaptada para a forma igualmente coloquial "Eu não posso 'pegar leve' comigo mesmo" para equivalência semântica. Além disso, a expressão "make excuses" foi adaptada para o gerúndio "ficar arranjando desculpas", pois essa expressão no português representa uma forma nominal comumente utilizada para expressar crítica a comportamentos protelatórios. No item 87 (I have rarely been able to stick to my resolutions), foram acrescentados os termos "ou projetos pessoais/profissionais" para auxiliar na compreensão da expressão "resoluções".

\section{Avaliação dos Juízes}

A concordância geral na avaliação os itens foi de $95,1 \%$, classificada como alta (Stemler, 2004). A concordância por aspecto avaliado também foi classificada como alta: 91,9\% para manutenção do conteúdo original; $97,1 \%$ para a clareza do item; e $96,3 \%$ para escrita neutra e sem regionalismos.

A partir dos comentários qualitativos dos juízes, foram alterados os itens $2,10,18,24,53$ e 59 . No item 2 (I find $m y$ self clinging to people I'm close to because I am afraid they'll leave me), o termo "clining" inicialmente foi traduzido como "grudo" e, após sugestão dos juízes, foi alterado para "agarro". No item 10 (I think thatif I do what I want, I'm only asking for trouble), a expressão "I do what I want" foi adaptada para "o que estou afim" e, posteriormente, alterada para "o que quero". A expressão "If I make a mistake" do item (If I make a mistake, I deserve to be punished) foi traduzida para "Se eu fizer algo errado" e modificada para "Se eu cometer algum erro".

No item 24 (I'm incompetent when it comes to achievement), a expressão "when it comes to achievement" foi adaptada para "para conquistar coisas importantes" e alterada para "quando se refere a realizações pessoais/profissionais". O item 36 (If I don't try my hardest, I should expect to lose out) o trecho "If I don't try my hardest", adaptado como "Caso não me esforce ao máximo naquilo que considero importante", foi modificado para "Se eu não der o meu melhor".

O item 53 (You can't be too careful; something will always go wrong) foi adaptado para "Cuidado nunca é demais; quase sempre algo ruim vai acontecer" e, após sugestões dos juízes, foi definido como "Todo cuidado é pouco; quase sempre algo vai dar errado". Após a adaptação do item 59 (I feel that I'm notlovable), foi inclusa a expressão entre parêntesis "(por causa dos meus defeitos)", com o intuito de explicar o item direcionando para o esquema de Defectividade, no entanto, a maior parte dos juízes sugeriu sua retirada.

\section{Avaliação pelo Público-Alvo}

Os participantes do grupo focal relataram que, em geral, as instruções e questões estavam claras e compreensíveis. No entanto, alguns participantes relataram dificuldades na compreensão de alguns itens do EID de Isolamento Social/Alienação, apontando para a necessidade de complementos. Os itens destacados foram "4. Não me encaixo", "22. Eu sou fundamentalmente diferente das outras pessoas" e " 40 . Eu não faço parte; sou uma pessoa solitária". No item 4 , foi relatado que 
poderia haver complemento para especificar "onde" o sujeito sente que não se encaixa. No item 22 , houve dúvida sobre a expressão "fundamentalmente". No item 40, foi relatada que seria interessante especificar em que o indivíduo consideraria "não faço parte".

Os comentários são pertinentes para a observação clínica e para a aplicação do questionário. As sugestões não geraram mudanças no instrumento com intuito de não alterar o conteúdo do item. Essa posição foi adotada para que o questionário seguisse o mais próximo possível do original.

\section{Retrotradução}

A retrotradução foi conferida e encaminhada ao ISST. Não foram solicitadas reformulações e a versão foi aprovada.

\section{Estudo Piloto}

Os participantes dessa etapa relataram que, em geral, não houve dificuldade na compreensão dos itens. Com exceção do item 4, "Não me encaixo", no qual alguns participantes relataram necessidade de complemento, bem como observado no grupo focal.

Tabela 1

Itens com Adaptações para Equivalência de Significado e Alterações após Avaliação dos Juízes

\begin{tabular}{|c|c|c|c|c|c|}
\hline Item & Item original & Adaptação final & Retrotradução & Esquema & Domínio \\
\hline 1 & $\begin{array}{l}\text { I haven't had someone to } \\
\text { nurture me, share him/ } \\
\text { herself with me, or care } \\
\text { deeply about everything } \\
\text { that happens to me. }\end{array}$ & $\begin{array}{l}\text { Eu não tive ninguém para } \\
\text { me dar afeto, cuidado e } \\
\text { proteção, que partilhasse } \\
\text { sua vida comigo, ou que } \\
\text { se importasse de verdade } \\
\text { com as coisas que me } \\
\text { acontecem. }\end{array}$ & $\begin{array}{l}\text { I have had no one to } \\
\text { nurture me, to share his } \\
\text { or her life with me or } \\
\text { who truly cared about } \\
\text { everything that happens } \\
\text { to me. }\end{array}$ & $\begin{array}{l}\text { Privação } \\
\text { emocional }\end{array}$ & $1^{\circ}$ \\
\hline 2 & $\begin{array}{l}\text { I find myself clinging } \\
\text { to people I'm close to } \\
\text { because I am afraid } \\
\text { they'll leave me. }\end{array}$ & $\begin{array}{l}\text { Eu percebo que me } \\
\text { agarro às pessoas que } \\
\text { são próximas de mim } \\
\text { com medo de que elas me } \\
\text { abandonem. }\end{array}$ & $\begin{array}{l}\text { I see myself clinging } \\
\text { to people I'm close to, } \\
\text { because I'm afraid they'll } \\
\text { leave me. }\end{array}$ & Abandono & $1^{\circ}$ \\
\hline 7 & $\begin{array}{l}\text { I do not feel capable of } \\
\text { getting by on my own in } \\
\text { everyday life. }\end{array}$ & $\begin{array}{l}\text { Eu não me sinto capaz de } \\
\text { me virar sem a ajuda dos } \\
\text { outros no dia a dia. }\end{array}$ & $\begin{array}{l}\text { I do not feel capable of } \\
\text { getting by without help } \\
\text { from others in everyday life. }\end{array}$ & $\begin{array}{l}\text { Dependência/ } \\
\text { Incompetência }\end{array}$ & $2^{\circ}$ \\
\hline 8 & $\begin{array}{l}\text { I can't seem to escape } \\
\text { the feeling that } \\
\text { something bad is about } \\
\text { to happen. }\end{array}$ & $\begin{array}{l}\text { Parece que eu não consigo } \\
\text { parar de sentir que } \\
\text { algo ruim está prestes a } \\
\text { acontecer. }\end{array}$ & $\begin{array}{l}\text { It seems that I can't help } \\
\text { feeling that something } \\
\text { bad is about to happen. }\end{array}$ & $\begin{array}{l}\text { Vulnerabilidade } \\
\text { ao dano ou à } \\
\text { doença }\end{array}$ & $2^{\circ}$ \\
\hline 10 & $\begin{array}{l}\text { I think that if I do what } \\
\text { I want, I'm only asking } \\
\text { for trouble. }\end{array}$ & $\begin{array}{l}\text { Eu sinto que, se eu fizer o } \\
\text { que quero, os outros não } \\
\text { me apoiarão e poderão } \\
\text { ficar bravos ou chateados } \\
\text { comigo. }\end{array}$ & $\begin{array}{l}\text { I feel that if I do what } \\
\text { I want, others will not } \\
\text { support me and may be } \\
\text { angry or upset with me. }\end{array}$ & Subjugação & $4^{\circ}$ \\
\hline 17 & $\begin{array}{l}\text { Even when things seem } \\
\text { to be going well, I feel } \\
\text { that it is only temporary. }\end{array}$ & $\begin{array}{l}\text { Mesmo quando as coisas } \\
\text { parecem estar indo bem, } \\
\text { eu sinto que é apenas } \\
\text { questão de tempo para } \\
\text { que elas comecem a dar } \\
\text { errado. }\end{array}$ & $\begin{array}{l}\text { Even when things seem } \\
\text { to be going well, I feel } \\
\text { it's just a matter of time } \\
\text { before they start to go } \\
\text { wrong. }\end{array}$ & $\begin{array}{l}\text { Negatividade/ } \\
\text { Pessimismo }\end{array}$ & $1^{\circ}$ \\
\hline 28 & $\begin{array}{l}\text { I feel as if I have no choice } \\
\text { but to give in to other } \\
\text { people's wishes, or else } \\
\text { they will retaliate, get } \\
\text { angry, or reject me in } \\
\text { some way. }\end{array}$ & $\begin{array}{l}\text { Eu sinto como se eu não } \\
\text { tivesse outra opção a não } \\
\text { ser ceder aos desejos dos } \\
\text { outros, se não eles irão } \\
\text { ficar bravos, me rejeitar ou } \\
\text { retaliar de alguma forma. }\end{array}$ & $\begin{array}{l}\text { I feel that I have no } \\
\text { choice but to give in to } \\
\text { other people's wishes, } \\
\text { otherwise they will get } \\
\text { angry at me, retaliate or } \\
\text { reject me in some way. }\end{array}$ & Subjugação & $4^{\circ}$ \\
\hline 32 & $\begin{array}{l}\text { I'm special and shouldn't } \\
\text { have to accept many } \\
\text { of the restrictions or } \\
\text { limitations placed on } \\
\text { other people. }\end{array}$ & $\begin{array}{l}\text { Eu sou diferenciado e não } \\
\text { deveria ter que aceitar } \\
\text { muitas das restrições ou } \\
\text { limitações impostas às } \\
\text { outras pessoas. }\end{array}$ & $\begin{array}{l}\text { I'm distinct and should } \\
\text { not have to accept many } \\
\text { of the restrictions or } \\
\text { limitations imposed on } \\
\text { other people. }\end{array}$ & $\begin{array}{l}\text { Merecimento/ } \\
\text { Grandiosidade }\end{array}$ & $3^{\circ}$ \\
\hline 36 & $\begin{array}{l}\text { If I don't try my } \\
\text { hardest, I should expect } \\
\text { to lose out. }\end{array}$ & $\begin{array}{l}\text { Se eu não der o meu } \\
\text { melhor, eu tenho que me } \\
\text { dar mal mesmo. }\end{array}$ & $\begin{array}{l}\text { If I don't do my best, I } \\
\text { have to really get burned. }\end{array}$ & Postura punitiva & $5^{\circ}$ \\
\hline
\end{tabular}


Tabela 1 (continuação)

Itens com Adaptações para Equivalência de Significado e Alterações após Avaliação dos Juízes

\begin{tabular}{|c|c|c|c|c|c|}
\hline Item & Item original & Adaptação final & Retrotradução & Esquema & Domínio \\
\hline 44 & $\begin{array}{l}\text { I worry about being } \\
\text { physically attacked by } \\
\text { people. }\end{array}$ & $\begin{array}{l}\text { Eu me preocupo muito em } \\
\text { ser agredido fisicamente } \\
\text { por alguém. }\end{array}$ & $\begin{array}{l}\text { I worry a lot about being } \\
\text { physically attacked by } \\
\text { someone. }\end{array}$ & $\begin{array}{l}\text { Vulnerabilidade } \\
\text { ao dano ou à } \\
\text { doença }\end{array}$ & $2^{\circ}$ \\
\hline 53 & $\begin{array}{l}\text { You can't be too careful; } \\
\text { something will always go } \\
\text { wrong. }\end{array}$ & $\begin{array}{l}\text { Todo cuidado é pouco; } \\
\text { quase sempre algo vai dar } \\
\text { errado. }\end{array}$ & $\begin{array}{l}\text { Being careful is never too } \\
\text { much; something will } \\
\text { almost always go wrong. }\end{array}$ & $\begin{array}{l}\text { Negatividade/ } \\
\text { Pessimismo }\end{array}$ & $1^{\circ}$ \\
\hline 59 & $\begin{array}{l}\text { I feel that I'm not } \\
\text { lovable. }\end{array}$ & $\begin{array}{l}\text { Eu sinto que sou alguém } \\
\text { que não pode ser amado. }\end{array}$ & $\begin{array}{l}\text { I feel that I am not a } \\
\text { lovable person. }\end{array}$ & $\begin{array}{l}\text { Defectividade e } \\
\text { vergonha }\end{array}$ & $1^{\circ}$ \\
\hline 63 & $\begin{array}{l}\text { I often feel as if my } \\
\text { parent(s) are living } \\
\text { through me - that I don't } \\
\text { have a life of my own. }\end{array}$ & $\begin{array}{l}\text { Eu frequentemente } \\
\text { sinto como se meus pais } \\
\text { vivessem a minha vida, } \\
\text { ou vivessem através de } \\
\text { mim - é como se eu não } \\
\text { tivesse uma vida própria. }\end{array}$ & $\begin{array}{l}\text { I often feel as if my } \\
\text { parent(s) were living my } \\
\text { life, or as if they were } \\
\text { living through me - as if } \\
\text { I didn't have a life of my } \\
\text { own. }\end{array}$ & $\begin{array}{l}\text { Emaranhamento/ } \\
\text { Self } \\
\text { Subdesenvolvido }\end{array}$ & $2^{\circ}$ \\
\hline 85 & $\begin{array}{l}\text { I can't let myself off the } \\
\text { hook easily or make } \\
\text { excuses for my mistakes. }\end{array}$ & $\begin{array}{l}\text { Eu não posso "pegar leve" } \\
\text { comigo mesmo ou ficar } \\
\text { arranjando desculpas } \\
\text { pelos meus erros. }\end{array}$ & $\begin{array}{l}\text { I can't go easy on myself } \\
\text { or make excuses for my } \\
\text { mistakes. }\end{array}$ & $\begin{array}{l}\text { Padrões } \\
\text { inflexíveis/ } \\
\text { Hipercriticismo }\end{array}$ & $5^{\circ}$ \\
\hline 87 & $\begin{array}{l}\text { I have rarely been able to } \\
\text { stick to my resolutions. }\end{array}$ & $\begin{array}{l}\text { Eu raramente consigo } \\
\text { seguir com minhas } \\
\text { resoluções ou projetos } \\
\text { pessoais/ profissionais. }\end{array}$ & $\begin{array}{l}\text { I have rarely been able } \\
\text { to stick to my resolutions } \\
\text { or personal/professional } \\
\text { projects. }\end{array}$ & $\begin{array}{l}\text { Autocontrole e } \\
\text { autodisciplina } \\
\text { insuficientes }\end{array}$ & $3^{\circ}$ \\
\hline
\end{tabular}

\section{Segunda Etapa: Avaliação Preliminar de Propriedades Psicométricas}

Participaram da segunda etapa 144 (72\%) sujeitos do sexo feminino e 56 (28\%) do sexo masculino, com média de idade de 30,94 anos $(D P=9,82)$. A escolaridade dos participantes foi ensino superior incompleto $(37,5 \%)$, ensino superior completo (22\%), pós-graduação completa incluindo stricto sensu e lato sensu (38\%), ensino médio incompleto e completo $(2,5 \%)$.
No que se refere à consistência interna global, considerando os 90 itens, o alfa de Cronbach foi 0,965 , considerado excelente. A confiabilidade por fator foi avaliada a partir de cada EID, considerando os cinco itens inclusos em cada um deles. Os resultados de confiabilidade por fator, listados na Tabela 2, foram considerados satisfatórios, com exceção do EID "Merecimento/ Grandiosidade”.

Tabela 2

Resultados da Fidedignidade por EID

\begin{tabular}{|c|c|}
\hline Esquema Inicial Desadaptativo & Alfa de Cronbach \\
\hline Privação emocional & 0,84 \\
\hline Abandono & 0,88 \\
\hline Desconfiança/Abuso & 0,83 \\
\hline Isolamento social/Alienação & 0,85 \\
\hline Defectividade/Vergonha & 0,85 \\
\hline Fracasso & 0,92 \\
\hline Dependência/Incompetência & 0,77 \\
\hline Vulnerabilidade ao dano e doença & 0,76 \\
\hline Emaranhamento & 0,80 \\
\hline Subjugação & 0,85 \\
\hline Autossacrifício & 0,78 \\
\hline Inibição emocional & 0,84 \\
\hline Padrões inflexíveis & 0,77 \\
\hline Arrogo/Grandiosidade & 0,67 \\
\hline Autocontrole/Autodisciplina insuficientes & 0,82 \\
\hline
\end{tabular}


Tabela 2 (continuação)

Resultados da Fidedignidade por EID

\begin{tabular}{|c|c|}
\hline Esquema Inicial Desadaptativo & Alfa de Cronbach \\
\hline Busca de aprovação/Reconhecimento & 0,83 \\
\hline Negatividade/Pessimismo & 0,77 \\
\hline Postura punitiva & 0,80 \\
\hline
\end{tabular}

\section{Discussão}

No que se refere ao processo de tradução e adaptação do instrumento, o YSQ-S3 apresenta bom ajuste semântico e está adequado para uso no Brasil. A concordância entre juízes foi classificada como alta, demonstrando a adequação do instrumento quanto à tradução, conteúdo, manutenção do significado e baixa presença de regionalismos. Com exceção de alguns itens do EID de Isolamento social, os participantes do grupo focal e do estudo piloto não apresentaram dificuldades na compreensão do instrumento. $\mathrm{Na}$ retrotradução, os itens mantiveram o conteúdo dos originais, e ela foi aceita sem necessidade de ajustes, o que permite evidenciar a qualidade da versão oficial em português brasileiro.

Os dados preliminares de fidedignidade por EID indicaram boa consistência interna, com resultados próximos aos apresentados nos estudos de outros países como a versão tailandesa (Sakulsriprasert et al., 2016), franco-canadense (Hawke, \& Provencher, 2012), romena (Trip, 2006), dinamarquesa (Bach et al., 2017a), polonesa (Oettingen et al., 2017) alemã (Kriston et al., 2013) e coreana (Lee et al., 2015). No que se refere ao EID de Arrogo/Grandiosidade, a consistência interna ligeiramente inferior às demais também foi constatada em outros estudos, como nas versões polonesa $(\alpha=0,62)$, na germânica $(\alpha=0,67)$, coreana $(\alpha=0,59)$ e franco-canadense $(\alpha=0,57)$. Esse dado referente ao Arrogo/Grandiosidade pode ser justificado pela variabilidade de conteúdo dos itens que mensuram esse EID. As questões contemplam aspectos referentes à sensação de ser especial e superior, bem como dificuldades em aceitar "não" e em respeitar regras. Esses conteúdos representam diferentes facetas do EID, mas, por vezes, podem não estar diretamente relacionados, o que pode levar à consistência interna ligeiramente inferior.

No que se refere às limitações, a adaptação dos itens pode ter a influência das características do grupo focal. $\mathrm{Na}$ etapa de avaliação preliminar de propriedades psicométricas, destaca-se o tamanho da amostra, em função da proposta de apresentação de dados preliminares. Além disso, cabe ressaltar a homogeneidade da amostra em relação ao sexo, predominando feminino, e escolaridade, com predominância acima de ensino superior completo. Portanto, há importância de futuros estudos com amostras mais heterogêneas. Destaca-se ainda a necessidade de análises estatísticas mais robustas para avaliação das demais propriedades psicométricas do instrumento. O projeto guarda-chuva no qual está inserida esta pesquisa contempla o seguimento de estudos complementares de fidedignidade com uma amostra maior, de evidências de validade e análise fatorial que serão publicados em momento posterior.

\section{Agradecimentos}

Agradecemos aos demais integrantes do Grupo de Avaliação e Atendimento em Psicoterapia Cognitiva e Comportamental (GAAPCC) e aos participantes voluntários da pesquisa. Além disso, agradecemos o apoio financeiro do CNPq.

\section{Financiamentos}

Todas as fontes de financiamento para elaboração e produção do estudo (coleta, análise e interpretação dos dados, bem como, escrita dos resultados no presente no manuscrito) foram fornecidas pelo projeto de pesquisa 'código do financiamento 433343/2018-4, agência de fomento CNPq'.

\section{Contribuições dos autores}

Declaramos que todos os autores participaram da elaboração do manuscrito. Especificamente, o(s) autor(es) Lauren Heineck de Souza e Elisa Steinhorst Damasceno participou(ram) da redação inicial do estudo - conceitualização, investigação, visualização, o(s) autor(es) Lauren Heineck de Souza, Elisa Steinhorst Damasceno e Margareth da Silva Oliveira participou(ram) da análise dos dados, e o(s) autor(es) Lauren Heineck de Souza, Elisa Steinhorst Damasceno, Felipe Gonçalves Ferronatto e Margareth da Silva Oliveira participou(ram) da redação final do trabalho revisão e edição.

\section{Disponibilidade dos dados e materiais}

Todos os dados e sintaxes gerados e analisados durante esta pesquisa serão tratados com total sigilo devido às exigências do Comitê de Ética em Pesquisa em Seres Humanos. Porém, o conjunto de dados e sintaxes que apoiam as conclusões deste artigo estão disponíveis mediante razoável solicitação ao autor principal do estudo.

\section{Conflito de interesses}

Os autores declaram que não há conflitos de interesses. 


\section{Referências}

Alfasfos L. (2009). The Early Maladaptive Schemas and their Correlations with the Psychiatric Symptoms and the Personality Accentuations for Palestinian Students (Doctoral dissertation). Recuperado de http://ediss.sub.uni-hamburg.de/volltexte/2010/4827/pdf/ohne_appendix_printed.pdf

Bach, B., Lockwood, G., \& Young, J. E. (2017b). A new look at the schema therapy model: organization and role of early maladaptive schemas. CognBehavTher, 19, 1-22. doi: 10.1080/1650603.2017.1410566

Bach, B., Simonsen, E., Christoffersen, P., \& Kriston, L. (2017a). The Young Schema Questionnaire 3 Short Form (YSQ-S3): Psychometric properties and association with personality disorders in a Danish mixed sample. European Journal of Psychological Assessment. 33(2), 134143. doi: 10.1027/1015-5759/a000272

Borsa, J. L., Damásio, B. F., \& Bandeira, D. R. (2012). Adaptação e validação de instrumentos psicológicos entre culturas: Algumas considerações. Paidéia, 22(53), 423-432. doi: 10.1590/S0103-863×2012000300014

Calvete, E., Orue, I., \& González-Diez, Z. (2013). An Examination of the Structure and Stability of Early Maladaptive Schemas by Means of the Young Schema Questionnaire - 3. European Journal of Psychological Assessment, 29(4), 283-290. doi: 10.1027/1015-5759/a000158

Cassepp-Borges, V., Balbinotti, M. A. A., \& Teodoro, M. L. M. (2010). Tradução e validação de conteúdo: Uma proposta para a adaptação de instrumentos. Em L. Pasquali (Ed), Instrumentação psicológica: Fundamentos e práticas (pp. 506-520). Porto Alegre: Artmed.

Cazassa, M. J., \& Oliveira, M. S. (2012). Validação brasileira do questionário de esquemas de Young: versão breve. Estudos de Psicologia (Campinas), 29(1), 23-31. doi: 10.1590/S0103-166X20120001000003

Edwards, D., \& Arntz, A. A. (2012). Schema therapy in historical perspective. Em M. van der Vreeswijk, J. Broersen, \& M. Nadort (Eds.), Handbook of Schema Therapy: Theory, Researchand Pratice (pp. 3-26). Chichester: Willey-Blackwell.

Hambleton, R. K. (2005). Issues, designs and technical guidelines for adapting tests into multiple languages and cultures. Em R. K. Hambleton, P. F. Merenda \& S. D. Spielberger (Eds), Adapting educational and psychological tests for cross-cultural assentment (pp. 3-38). Nova Jersey: Lawrence Erlbaum.

Hawke, L. D., \& Provencher, M. D. (2011). Schema theory and schema therapy in mood and anxiety disorders: A review. JCognPsychother, 25, 257-276. doi: 10.1891/0889-8391.25.4.257

Hawke, L. D., \& Provencher, M. D. (2012). The Canadian French Young Schema Questionnaire: Confirmatory Factor Analysis and Validation in Clinical and Nonclinical Samples. Canadian Journal of Behavioural Science. 44(1), 40-49. doi: 10.1037/a0026197

Jacob, G. A., \& Arntz, A. (2013). Schema therapy for personality disorders - A review. International Journal of Cognitive Therapy, 6, $171-185$. doi:10.1521/ijct.2013.6.2.171

Kriston, L., Schäffer, J., Jacob, G. A., Härter, M., \& Hölzel, L. P. (2013). Reliability and validity of the German Version of the Young Schema Questionnaire-Short. European Journal of Psychological Assessment, 29(3), 205-212. doi: 10.1027/1015-5759/a000143

Lavergne, F., Marie, N., \& Mehran, F. (2015). Les 5 dimensions psychiques identifiées à partir des schémas précoces inadaptés: analyses statistiques du questionnaire des schémas (QSY-s3). L'Encéphale, 41(4), 314-322. doi: 10.1016/j.encep.2014.08.005

Lee, S. J., Choi, Y. H., Rim, H. D., Won, S. H., \& Lee, D. W. (2015). Reliability and Validity of the Korean Young Schema QuestionnaireShort Form-3 in Medical Student. Psychiatry Investigation,, 12(3), 295-304. doi: 10.4306/pi.2015.12.3.295

Lockwood, G., \& Perris, P. (2012). A new look at core emotional needs. Em M. van der Vreeswijk, J. Broersen \& M. Nadort (Eds.), Handbookof Schema Therapy: Theory, Research and Pratice (pp. 3-26). Chichester: Willey-Blackwell.

Lyrakos, D. G. (2014). The validity of Young Schema Questionnaire 3rd Version and the Schema Mode Inventory 2nd Version on the Greek population. Psychology, 5, 461-477. doi: 10.4236/psych.2014.5505

Oettingen, J., Chodkiewicz, J., Macik, D., \& Gruszcyńzka, E. (2017). Polish adaptation of the Young Schema Questionnaire 3 Short Form (YSQ-S3-PL). Psychiatria Polska, 82(1), 1-12. doi: 10.12740/PP/OnlineFirst/76541

Primi, R. (2003). Inteligência: Avanços nos modelos teóricos e nos instrumentos de medida. Avaliação Psicológica, 2, 67-77. Recuperado de http://pepsic.bvsalud.org/scielo.php?script=sci_arttext\&pid=S1677-04712003000100008\&lng=pt\&nrm =iso

Primi, R. (2010). Avaliação psicológica no Brasil: Fundamentos, situação atual e direções para o futuro. Psicologia: Teoria e Pesquisa, 26(spe), 25-35. doi: 10.1590/S0102-37722010000500003

Rijo, D, \& Gouveia, P. (2008). A confirmatory factor analysis study of the YSQ-S3 in a large Portuguese sample. Paper presented at the 3rd Annual International Society of Schema Therapy Meeting, Coimbra, Portugal.

Saariaho, T., Saariaho, A., Karila, I., \& Joukama, M. (2009). The psychometric properties of the finnish Young schema questionnaire in chronic pain patients and a non-clinical sample. Behavior Therapy and Experimental Psychiatry, 40(1), 158-68. doi: 10.1016/j.jbtep.2008.07.005

Sakulsriprasert, C., Phukao, D., Kanjanawong, S., \& Meemon, N. (2016). The reliability and factor structure of Thai young schema questionnaire-short form 3. Asian Journal of Psychiatry, 24, 85-90. doi:10.1016/j.ajp.2016.09.011

Saritas, D., \& Gençöz, T. (2011). Psychometric properties of "Young Schema Questionnaire-short form 3" in a Turkish adolescents sample. Journal of Cognitive and Behavioral Psychotherapies, 11(1), 83-96.

Schmidt, N. B., Joiner, T. E., Young, J. E., \& Telch, M. J. (1995). The Schema Questionnaire: Investigation of psychometric properties and the hierarchical structure of a measure of maladaptive schemas. Cognitive Therapy and Research, 19, 295-321. doi: 10.1007/BF02230402

Soygüt, G., Karaosmanoğlu, A., \& Cakir, Z. (2009). Assessment of early maladaptive schemas: A psychometric study of the Turkish Young Schema Questionnaire-Short Form-3. Türk Psikiyatri Dergisi, 20(1), 1-10. Recuperado de http://www.turkpsikiyatri.com/C20S1/ en/75-84.pdf

Stemler, S.E. (2004). A comparison of consensus, consistency, and measurement approaches to estimating interrater reability. PractivalAssessment, Reserch \& Evaluation, 9(4), 1-11. Recuperado de https://scholarworks.umass.edu/cgi/viewcontent.cgi? article $=1137 \&$ context $=$ pare

Taylor, C. D. J., Bee, P., \& Haddock, G. (2016). Does schema therapy change schemas and symptoms? Asystematic review across mental health disorders. Psychology and Psychotherapy: Theory, Research and Practice, 90(3), 456-479. doi: 10.1111/papt.12112

Trip, S. (2006). The Romanian a version of Young Schema Questionnaire-Short Form3 (YSQ-S3). Journal of Cognitive and Behavioral Psychotherapies, 6(2), 173-181. Recuperado de http://jebp.psychotherapy.ro/vol6no2/the-romanian-version-of-young-schemaquestionnaire-short-form-3-ysq-s3/

Van Vreeswijk, M., Broersen, J., \& Nadort, M. (2012). The Wiley-Blackwell handbook of schema therapy: Theory, research and practice. Chichester, UK: Wiley-Blackwell.

Young, J. E. (1990). Cognitive Therapy for personality disorders: A schema-focused approach (rev. ed.). Sarasota, FL: Professional Resources Press. 
Young, J. E. (2003). Young Schema Questionnaire - Long form 3 (YSQ-L3). New York: Cognitive Therapy Center of New York. Young, J. E. (2005). Young Schema Questionnaire - Short form 3 (YSQ-S3). New York: Cognitive Therapy Center of New York. Young, J. E. (2014). Early maladaptative schemas - Revised. New York, NY: Cognitive Therapy Center of New York.

Young, J. E., \& Brown, G. (1990). Young Schema Questionnaire. New York: Cognitive Therapy Center of New York.

Young, J. E., \& Brown, G. (1999). Young schema questionnaire: Short version. New York: Cognitive Therapy Center of New York.

Young, J. E., Klosko, J. S., \& Weishaar, M. E. (2003). Schema Therapy: A Practitioners Guide. New York: Guilford Press.

\section{Sobre os autores}

Lauren Heineck de Souza é psicóloga e mestranda na Pontifícia Universidade Católica do Rio Grande do Sul (PUCRS).

Elisa Steinhorst Damasceno é psicóloga e mestranda na Pontifícia Universidade Católica do Rio Grande do Sul (PUCRS).

Felipe Gonçalves Ferronatto é graduando em Psicologia e bolsista de Iniciação Científica na Pontifícia Universidade Católica do Rio Grande do Sul (PUCRS).

Margareth da Silva Oliveira é psicóloga, doutora em Psiquiatria e Psicologia Médica pela Universidade Federal de São Paulo. Professora de Pós-Graduação em Psicologia da Pontifícia Universidade Católica do Rio Grande do Sul (PUCRS) e coordenadora do Grupo de Pesquisa, Avaliação e Atendimento em Psicoterapias Cognitivas e Comportamentais (GAAPCC).

\section{Como citar este artigo}

Souza et al. (2020). Adaptação Brasileira do Questionário de Esquemas de Young - Versão Breve (YSQ-S3). Avaliação Psicológica, 19(4), 451-460. http://dx.doi.org/10.15689/ap.2020.1904.17377.11 